\title{
Preemption Window for Burst Differentiation in OBS
}

\author{
Miroslaw Klinkowski ${ }^{1,2)}$, Davide Careglio ${ }^{1)}$, Daniel Morató ${ }^{3)}$ and Josep Solé-Pareta ${ }^{1)}$ \\ 1) Universitat Politècnica de Catalunya, Jordi Girona, 1-3, 08034 Barcelona, Catalunya, Spain \\ 2) National Institute of Telecommunications, 1 Szachowa Street, 04-894 Warsaw, Poland \\ ${ }^{3)}$ Public University of Navarra, Campus de Arrosada, 31006, Pamplona, Spain \\ \{mklinkow,careglio,pareta\}@ac.upc.edu,daniel.morato@unavarra.es
}

\begin{abstract}
This paper presents a novel control architecture for optical burst switching networks to efficiently apply burst preemption without the resources overbooking, which is specific to conventional OBS. Simulation results prove the effectiveness of this proposal.

(C)2007 Optical Society of America

OCIS codes: (060.4250) Networks, (060.4259) Networks, packet-switched
\end{abstract}

\section{Introduction}

Optical Burst Switching (OBS) is the most promising photonic network architecture to cope with an efficient transport of IP traffic [1]. In conventional OBS the packets from the access networks are aggregated and assembled into large data bursts at the edge nodes. Meanwhile, the control information is transmitted out-of-band and delivered with some offset time prior to the data burst in such a way that the intermediate nodes have enough time, both to process this information and to reconfigure the switching matrix.

Several strategies have been considered to provide contention resolution with burst differentiation. The most effective solutions are based on burst preemption. In case of contention, it allows the switch controller to overwrite a Low Priority (LP) reservation with a later arriving High Priority (HP) one. Such preemption mechanism is used in QoS provisioning [2] as well as in redundant [3] and deflection routing. The general drawback of preemption -referred hereafter as Classical Preemption (CP)- is due to the so called phantom bursts: in case of successful preemption, the control packet corresponding to the preempted burst continues its travel to the destination node reserving resources at each downstream node of the path. This problem may lead to high network wastage and control processing effort under moderate and high traffic loads.

In this paper we propose a control architecture that easily overcomes this problem. The architecture assumes delaying the burst by means of an additional fiber delay coil introduced in the input ports of the core node as a substitution for the offset time introduced by the edge node. Besides compensating both control data processing and switching configuration times, this fiber provides an additional time window in which preemption is allowed. We show that the proposed preemption window mechanism, which expands look-ahead processing window techniques to the burst preemption context, achieves the performance of the classical burst preemption and at the same time avoids the presence of the phantom bursts.

\section{Preemption Window Mechanism}

We consider the OBS architecture presented in Fig. 1 with additional fiber delay coil -which are now commercially available and adopted in several test-beds as e.g. in [4]- inserted in the data path at the input ports of the core nodes. In such architecture there is no offset time set up by edge nodes. Control packet and burst travel simultaneously through the network (timely separated by the switching time, for simplicity intentionally omitted in Fig. 1). When both reach a core node, the control packet goes directly to the control unit, whilst the burst is delayed in the fiber delay coil by period $t_{p}$ (the processing time) and $T$ (the Preemption Window, PW). During $T$ the control unit can preempt a reservation by one with higher priority. The important rule of the $\mathrm{PW}$ mechanism is that the control packet, after its processing, is waiting for its burst in the memory of the control unit until $T$ expires and then they are either sent together to the next node (if the burst has not been preempted) or dropped (in case of successful preemption). After the control packet is sent, burst preemption is not allowed in the node. Thanks to these rules any control packet has its corresponding burst (no phantom bursts are present) and there is no need for any signaling procedure to be carried out in order to release the resources on the outgoing path in case of successful burst preemption.

The proposed preemption window can be also provided in conventional way, in the edge node as an additional offset, which accounts the windows in all the nodes of the path. A disadvantage of this solution is the increase of variation of offset times what further aggravates the unfairness in access to transmission resources [5]. 


\section{JWA85.pdf}

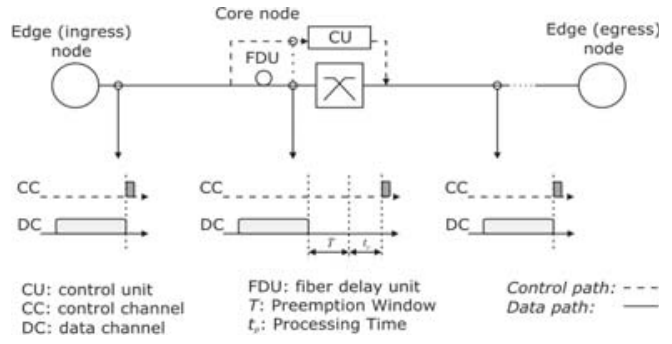

Fig 1. OBS architecture with preemption window.

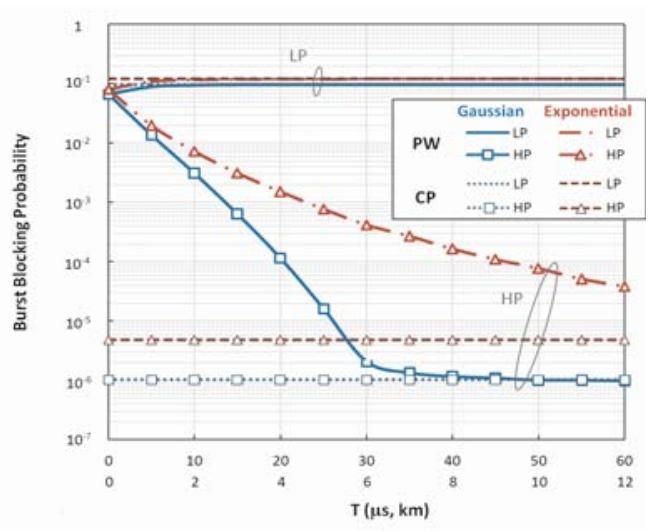

Fig 2. Burst blocking probability as a function of $T$ comparing Gaussian and Exponential traffic models $(\alpha=30 \%, \rho=0.8, W=16)$.

On the other hand, the application of OBS architecture with the offsets inserted in the core nodes brings additional benefits: 1) dismissing the offset variations (without using additional signaling overhead as in [6]), 2) reducing the edge buffer requirements since, once a burst is assembled, edge node can send it immediately, and 3) availing alternative routing since the paths can be created freely inside the network without any constraints in the number of hops due to the pre-calculated offset-time. Finally, if the nodes are bufferless, the scheduling complexity is relaxed since void-filling enhancement is not needed; indeed all offset-times are equal and each burst arrives after the previous one preventing the creation of voids between them. In [5] we demonstrate that this architecture performs as the conventional one in terms of delay, throughput and burst blocking probability.

In the context of burst differentiation, the value of $T$ becomes an important trade-off between high burst delay (too large PW) and ineffective burst preemption (too short PW). Scope of the next section is to determine the minimum value of $T$ that provides optimal blocking probability.

\section{Evaluation}

\subsection{Simulation scenario}

We are interested in evaluating the PW behavior thus a single bufferless OBS node with wavelength conversion, $4 \times 4$ input/output ports and operating at $10 \mathrm{Gbps}$ is considered (evaluating network performance is out of the scope of this letter). The LAUC (Latest Available Unused Channel) scheduling [7] with full preemption is applied. For lake of simplicity processing and switching times are set to 0 . The traffic is uniformly distributed between all ports. We consider two traffic models: a general Exponential and a specific Gaussian burst length and inter-arrival time distributions; the latter represents the traffic generated by a mixed time-length burstifier [8]. Both models use 40 kbytes $(32 \mu \mathrm{s})$ as mean bursts length; for Gaussian model we set up the standard deviation to $2 \mu \mathrm{s}$, and minimum and maximum burst lengths to 4 kbytes and 4 Mbytes, respectively. The mean burst inter-arrival times depends on the offered load $\rho$. The HP burst traffic ratio over overall one is denoted as $\alpha$. All the simulation results have $99 \%$ level of confidence.

\subsection{Numerical results}

In Fig. 2, we firstly compare the Classical Preemption (CP) with our Preemption Window (PW) solution as a function of the delay $T$. When $T=0$, there is no possibility of preemption and PW performs as a simple scheduling without burst differentiation. When $T$ increases, HP (LP) burst blocking probability decreases (increases) and approximates to an asymptote, which corresponds to the results obtained with CP. In case of Gaussian traffic, PW quickly reaches the CP performance ( $T$ larger than $30 \mu \mathrm{s})$, while worse results are obtained with Exponential one ( $T$ larger than $60 \mu \mathrm{s})$. This is because the former generates a concentration of burst durations more closed to the length of the fiber delay coil than latter; it has to be underlined that this Gaussian traffic model can be easily obtained well tuning the time/length thresholds of the burstifier [8]. 


\section{JWA85.pdf}

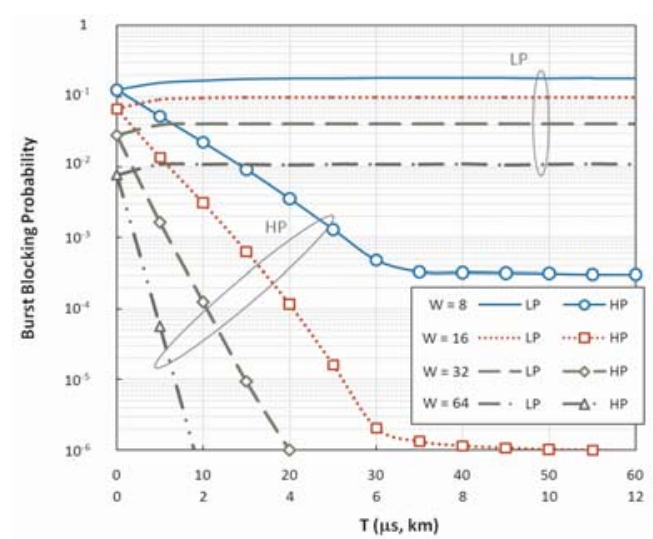

Fig 3. Burst blocking probability as a function of $T$, and of $W(\alpha=30 \%, \rho=0.8$, Gaussian traffic model $)$

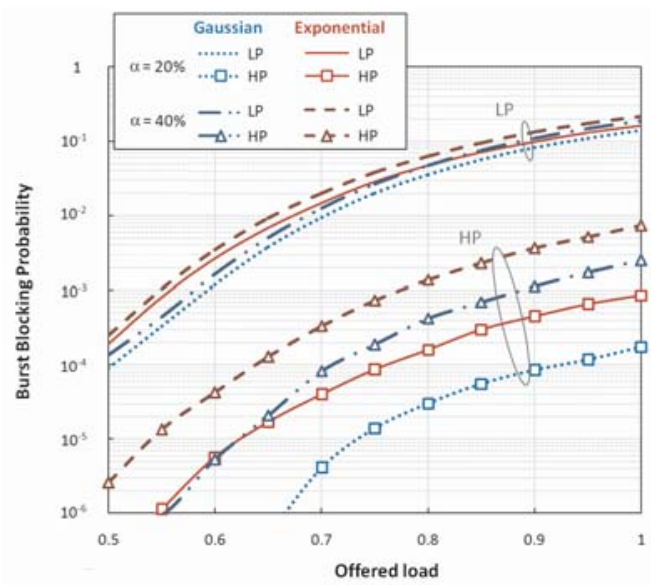

Fig 4. Burst blocking probability as a function of $\rho$ comparing Gaussian and Exponential traffic models and different $\alpha(T=10 \mu$ s and $W=32)$.

As Fig. 3 shows, burst blocking probability would be further reduced in the systems with more wavelengths. We can discern that for $T \geq 30 \mu \mathrm{s}(6 \mathrm{~km})$ and $\mathrm{W} \geq 16$ wavelengths, HP burst blocking probability is less than $10^{-6}$.

In Fig. 4, we analyze the blocking probability as a function of the offered load and of the percentage of HP burst traffic load. The $T$ window is fixed to $10 \mu \mathrm{s}(2 \mathrm{~km})$ and 32 wavelengths are considered. We can observe that PW achieves very low HP burst blocking probabilities, e.g. $10^{-5}$ at $\rho=0.65$ and $\alpha=40 \%$. Again, PW behaves better when the burst generation follows the Gaussian model.

\section{Conclusions}

In this paper we propose a dedicated control architecture for burst preemption in OBS networks. A preemption window is achieved using fiber delay coil at the input of the core nodes. Thanks to this window the problem of phantom bursts disappears without the need of additional mechanisms.

Simulation results show that the PW mechanism achieves the same performance of the conventional preemptive scheme. The obtained values show the feasibility of its application; e.g., a fiber of about $6 \mathrm{~km}$ is enough when a Gaussian distributed burst traffic model is applied.

Furthermore, the PW mechanism can be also used in a conventional OBS network as well as with any other preemptive technique like burst segmentation.

\section{References}

[1] C. Qiao and M. Yoo, "Optical burst switching (OBS) - a new paradigm for an optical Internet," J. High Speed Networks, vol. 8, no. 1, Mar. 1999, pp. 69-84.

[2] V. M. Vokkarane and J. P. Jue, "Prioritized burst segmentation and composite burst-assembly techniques for QoS support in optical burst switched networks," IEEE J. Select. Areas Commun., vol. 21, no. 7, Sep. 2003, pp. 1198-1209.

[3] J. Li and K. L. Yeung, "Burst cloning with load balancing", Proc. OFC 2006, Anaheim, CL, Mar. 2006.

[4] A. Al Amin et al., "40/10 gbps bit-rate transparent burst switching and contention resolving wavelength conversion in an optical router prototype", Proc. ECOC, Cannes, Oct. 2006.

[5] M. Klinkowski, D. Careglio, and J. Solé-Pareta, "Offset-time emulated OBS control architecture," Proc. ECOC 2006, Cannes, France, Oct. 2006.

[6] N. Barakat and E.H. Sargent, "Dual-header optical burst switching: a new architecture for WDM burst-switched networks," Proc. Infocom 2005, Miami, FL, Mar. 2005.

[7] Y. Xiong et al, "Control architecture in optical burst-switched WDM networks," IEEE J. Select. Areas Commun., vol. 18, no. 10, Oct. 2000, pp. 1838-1851.

[8] X. Yu et al, "Traffic statistics and performance evaluation in optical burst switched networks," IEEE/OSA J. Lightwave Technol., vol. 22, no. 12 , Dec. 2004 , pp. $2722-2738$. 